\title{
Join the dialogue
}

\section{The nanotoxicology community should implement guidelines on the types of information that are required in their research articles to improve the quality and relevance of the published papers.}

In the past decade, the number of published papers in the field of nanotoxicology - the study of the toxicity, and environmental, health and safety issues of nanomaterials has grown by nearly $600 \%$ (ref. 1). Most of these papers report in vitro studies that examine the toxicity of various nanomaterials. The studies are performed by delivering a certain amount of nanomaterial onto cells growing at the bottom of a culture plate and measuring how they respond. So much seems to have been done - using different model systems and nanomaterials - and yet, there are grumbles throughout the literature about the slow progress $^{2}$, misconceptions in and of the field ${ }^{3}$, and proposals on what the community needs to do as a whole for the field to progress faster ${ }^{4}$. One thing is at least clear for now: few studies offer consistent results that are of value, and it is difficult to compare studies because they are often carried out using poorly characterized nanomaterials and arbitrary experimental conditions.

\section{"Materials characterization data is not a stand-alone piece of information."}

At Nature Nanotechnology, we have received a number of proposals asking for these concerns to be aired, for editors to implement guidelines and/or requirements for reporting nanotoxicology research and to actively enforce these. It is becoming clear, particularly in the bioscience and clinical research communities ${ }^{5}$, that good reporting has many benefits: it is essential for peer review, valuable for informing policy and future science, and above all, important for ensuring that money invested in research produces useful results ${ }^{6}$. As Kilkenny et al. have put it ${ }^{5}$, "failure to describe research methods and to report the results appropriately has potential scientific, ethical and economic implications for the entire research process and the reputation of those involved in it."

The most common question raised in nanotoxicology is whether there is a minimum set of physical and chemical characterization data for nanomaterials that is required for publication. Should this set contain the nanomaterial size, chemical composition, surface area and/or shape plus others? What is a reasonable number of parameters that should be included so that the study can be understood, and is reproducible and reliable, without putting a strain on budgets? Different proposals have been made in various publications: one had a list of 17 parameters outlining what is essential, valuable-but-not essential, or not significant ${ }^{7}$; another listed six items along with the challenges for obtaining them ${ }^{4}$; and another had eight must-have parameters ${ }^{2}$. In a Commentary in Nature Nanotechnology, Schururs and Lison ${ }^{8}$ point out that "characterization data is not a stand-alone piece of information" and that "the parameters examined should be those useful for analysing the results of the study and for understanding the mechanisms of toxicity".

Our position is that materials characterization should be done based on relevance to the study, but there are a number of common parameters that should be included in all papers. Various working groups involved in doing this seem to be narrowing the list down to: particle size and distribution, chemical composition, impurities, degree of nanomaterial aggregation or agglomeration under the experimental conditions, surface chemistry, surface area, morphology, surface reactivity and persistence. There are, of course, differing opinions on what this list should contain and whether such a list is necessary. However, for the field to progress, the community must start somewhere by agreeing on a basic list soon, and implementing it diligently.

Apart from the materials side of things, careful reporting of the experimental conditions (for example, the composition of biomolecules in the cell culture media) is equally important. More and more studies point to the significance of the proteins that adsorb on the nanoparticle surface when it comes into contact with the physiological environment or serum-containing cell culture media. These proteins are thought to 'passivate' the high surface energy of nanoparticles, and depending on the amount of serum present in the culture media and the total surface area of the nanoparticles, cells can interact and take up the nanoparticles in different ways. For these reasons, there is a need to define the nanomaterial along with the environment it is present in ${ }^{9}$.

\section{There is a need to define the nanomaterial along with the environment it is present in.}

Journal editors can draft guidelines and reject papers that do not meet the requirements, but ultimately, the responsibility and discipline for implementing them rests on the researchers. The big challenges in the coming years remain the same: to produce research results that can inform the public on the benefits and emergent risks of using nanomaterials.

Nature Nanotechnology is committed to supporting the community to implement guidelines. To start with, an open consultation and dialogue will be held at the upcoming 6th International Conference on Nanotoxicology in Beijing from 4 to 7 September 2012 (ref. 10). The aim of this two-hour session is to consult the community so that a formal set of characterization requirements for reporting nanotoxicology papers can be established. Nature Nanotechnology will offer to disseminate this information and/or revise our Guide to Authors. Please join the dialogue. Written comments and feedback are also welcomed at naturenano@nature.com (closing date: 30 November 2012).

\footnotetext{
References

1. Ostrowski, A. D., Martin, T., Conti, J., Hurt, I. \& Harthorn, B. H. J. Nanopart. Res. 11, 251-257 (2009)

2. Krug, H. F. \& Wick, P. Angew. Chem. Int. Ed. 50, 1260-1278 (2011).

3. Warheit, D. B. Nano Lett. 10, 4777-4782 (2010).

4. Bouwmeester, H. et al. Nanotoxicology 5, 1-11 (2011).

5. Kilkenny, C., Browne, W. J., Cuthill, I. C., Emerson, M. \& Altman, D. G. PLoS Biol. 8, e1000412 (2010).

6. Chalmers, I. \& Glasziou, P. Lancet 374, 86-89 (2009).

7. Oberdörster, G. et al. Part. Fibre Toxicol. 2, 1-35 (2005).

8. Schrurs, F. \& Lison, D. Nature Nanotech. 7, 546-548 (2012).

9. Lesniak, A. et al. ACS Nano 6, 5845-5857 (2012)

10. http://english.nanoctr.cas.cn/nanotoxicology2012
}

Published online: 19 August 2012 